\title{
Güçlü Direkt Radikal Tümlenmiş Modüllerin İki Yeni Genelleştirmesi
}

\section{Esra ÖZTÜRK SÖZEN*11]}

${ }^{1}$ Sinop Üniversitesi, Fen Edebiyat Fakültesi, Matematik Bölümü, 57000, Sinop, Türkiye

(Alınış / Received: 27.10.2020, Kabul / Accepted: 15.02.2021, Online Yayınlanma / Published Online: 15.04.2021)

\section{Anahtar Kelimeler}

Radikal tümlenmiş modül,

Güçlü $\oplus$ - $\delta$-radikal,

Tümlenmiş modül,

$\delta$-Küçük alt modül
Özet: Bir $A$ modülünün $\delta$-radikali $A$ nın direkt toplam terimi olacak şekilde bir $\delta$ tümleyene sahipse $A$ ya $\oplus$ - $\delta$-radikal tümlenmiş modül denir. Eğer $A$ nın $\delta$ radikalini içeren her alt modülü $A$ nın direkt toplam terimi olacak şekilde bir $\delta$ tümleyene sahip ise $A$ ya güçlü $\oplus-\delta$-radikal tümlenmiş modül adı verilir. Bu çalışmada tanımlanan bu modüllerin temel özellikleri araştırılmış, halka karakterizasyonları incelenmiștir. Özel olarak $R$ ayrık değerlendirme halkası üzerinde $\operatorname{Rad}(A) \ll A$ koşulunu gerçekleyen bir $A$ modülünün güçlü- $\delta$-radikal tümlenmiş olması için gerekli ve yeterli koşul $P, R$ nin maximal ideali; $K, R$ nin kesir cismi ve $Q=K / R$ olmak üzere $A=R^{x} \oplus K^{y} \oplus Q^{z} \oplus B_{P}(1,2, \ldots n)$ olacak şekilde $x, y, z, n \in \mathbb{N}$ mevcuttur olması ile verilir.

\section{Two New Genaralizations of Strongly Direct Radical Supplemented Modules}

\section{Keywords}

Radical supplemented module, Strongly $\oplus-\delta$-radical, Supplemented module, $\delta$-Small submodule

\begin{abstract}
A}$ module $A$ is called $\oplus-\delta$-radical supplemented if $\delta(A)$ has a $\delta$ supplement in $A$ that is a direct summand of $A$. A module $A$ is called strongly $\oplus-\delta$ radical supplemented if every submodule of $A$ containing $\delta(A)$ has a $\delta$-supplement in $A$ that is a direct summand of $A$. In this paper we investigate basic properties of these modules and obtain a characterization for $\delta$-semiperfect rings. In particular, a module $A$ with $\operatorname{Rad}(A) \ll A$ over a discrete valuating $\operatorname{ring} R$, is strongly $\oplus-\delta$ radical supplemented if $A=R^{x} \oplus K^{y} \oplus Q^{z} \oplus B_{P}(1,2, \ldots . n)$ for $x, y, z, n \in \mathbb{N}$ and the maximal ideal $P$ of $R$ where $K$ is the quotient field of $R$ and $Q=K / R$.
\end{abstract}

\section{Giriș}

Bu çalışmada $R$ ile birleşmeli ve birimli halkalar; $A$ ile de üniter $R$-modüller gösterilecektir. Ayrıca " $B \leq$ $A, B \unlhd A$ ve $A \ll B$ " notasyonları ile sirasiyla $B \operatorname{nin}, A$ nın "alt modülü, büyük alt modülü ve küçük alt modülü" olduğu ifade edilecektir. $A$ nin $B$ alt modülünün, $A$ nın sıfır alt modülü haricindeki alt modülleri ile arakesiti sıfirdan farklı ise $B$ ye $A$ nın büyük alt modülü denir. Diğer taraftan $A$ nın her $C$ öz alt modülü için $B+C \neq A$ oluyorsa $B$ ye $A$ nın küçük alt modülü denir. $A$ nın tüm küçük alt modüllerinin toplamı " $\operatorname{Rad}(A)$ " ile gösterilir. $B \leq A$ için $B+C=A$ koşulunu gerçekleyen $A$ nın alt modüllerinden minimal olanı $C$ ise, $C$ ye $B$ nin $A$ da bir tümleyeni denir. $\mathrm{Bu}$ ise $B+C=A$ ve $B \cap C \ll A$ olmasina eşdeğerdir. $A$ modülünün her alt modülü tümleyene sahipse $A$ ya tümlenmiş modül denir [1].

Zöschinger tümlenmiş modülleri zayıflatarak radikal tümlenmiş modülleri şu şekilde tanımlamıștır $[2,3]$ : $\operatorname{Rad}(A) \leq A, A$ da tümleyene sahip ise $A$ ya radikal tümlenmiş modül denir. 1990 yılında Muhammed ve Müller ise tümlenmiş modülleri kuvvetlendirerek, her alt modülü direkt toplam terimi olacak şekilde tümleyene sahip modülleri $\oplus$-tümlenmiş modüller olarak karakterize etmiştir [4].

Bir $A$ modülü için $Z(A)=\{a \in A \mid A n n(a) \unlhd R\}$ ile tanımlı olmak üzere, $Z(A)=A$ ise $A$ ya tekil modül; $Z(A)=0$ ise $A$ ya tekil olmayan modül denir. Zhou küçük alt modüllerin genellemesi olan $\delta$-küçük alt modülleri şu şekilde tanımlamıştır [5]: $\frac{A}{C}$ tekil olacak şekilde $A$ nın her $C$ öz alt modülü için $B+C=A$ ise $B$ ye $A$ nın $\delta$-küçük alt modülüdür denir ve " $B \ll_{\delta} A$ " ile gösterilir. $A$ nın tüm $\delta$-küçük alt modüllerinin toplamı $\delta(A)$ ile gösterilir. Ayrıca $\operatorname{Rad}(A) \leq \delta(A)$ olduğu açıktır. $\delta$-radikali kendisine eşit modüllere $\delta$-radikal modül denir [6]. Bundan yola çlkarak Koşan bir modülün $\delta$-tümleyen alt modüllerini tanımlayarak her alt modülünün $\delta$-tümleyeni mevcut olan modülleri $\delta$-tümlenmiş modül olarak tanımlamıştır [7]. Yani her $B \leq A$ için $B+C=A, B \cap$ $C \ll_{\delta} C$ olacak şekilde $C \leq A$ varsa $A$ ya $\delta$-tümlenmiş modül denir. Ayrıca, her alt modülü direkt toplam terimi olacak şekilde $\delta$-tümleyene sahip modüller $\oplus$ $\delta$-tümlenmiş modül olarak tanımlanmıştır [8]. 
Büyükaşık ve Türkmen radikali içeren her alt modülü tümleyene sahip modülleri güçlü radikal tümlenmiş modül olarak tanımlamıșlardır [9]. Nişancı Türkmen ve Pancar sırasıyla radikali, direkt toplam terimi olacak şekilde tümleyene sahip modülleri $\bigoplus$-radikal tümlenmiş modüller; radikali içeren her alt modülü direkt toplam terimi olacak şekilde tümleyene sahip modülleri güçlü $\oplus$-radikal tümlenmiş modüller olarak tanımlamışlardır, [10]. Eryılmaz $\delta$-radikali içeren her alt modülü (zayıf) $\delta$ - tümleyene sahip modülleri güçlü genelleștirilmiş (zayıf) $\delta$-tümlenmiş modül olarak tanımlamıştır, [11].

$\mathrm{Bu}$ çalışmada ise $\delta$-radikali ve $\delta$-radikali içeren her alt modülü direkt toplam terimi olacak şekilde $\delta$ tümleyen sahip olan modüller tanımlanmıştır. Böylece Nişancı Türkmen ve Pancar tarafından tanıtılan modüllerin yeni bir genellemesi elde edilmiştir. Bu modüller arasındaki kapsama ilişkin öz olduğunu gösteren örneklemelere yer verilmiştir. Sonlu sayıda (güçlü) $\oplus-\delta$-radikal tümlenmiş modülün toplamının da (güçlü) $\oplus-\delta$ - radikal tümlenmiş olduğu gösterilmiştir. Güçlü $\oplus-\delta$ - radikal tümlenmiş modüllerin alt modüllerinin ve faktör modüllerinin sınıfının hangi şartlarda korunduğu irdelenmiştir. Özel olarak elde edilen diğer önemli bulguların bir sıralamasını şu şekilde sunabiliriz. Direkt toplam terimi özelliğine sahip bir $A$ modülü güçlü $\oplus$ - $\delta$-radikal tümlenmiş ise $A$ dual sonlu $\oplus-\delta$ tümlenmiş modüldür. Direkt toplam terimi özelliğine sahip bir $R$ halkasının $\delta$-yarımükemmel olması için gerekli ve yeterli koşul her sonlu üretilmiş serbest $R$ modülün güçlü $\oplus-\delta$-radikal tümlenmiş olmasıdır. Ayrıca lokal Dedekind bölgesi üzerindeki bir $A$ modülünün güçlü $\bigoplus$ - $\delta$-radikal tümlenmiş olması için gerekli ve yeterli koşul $P, R$ nin maximal ideali; $K, R$ nin kesir cismi ve $Q=K / R$ olmak üzere $A=R^{x} \oplus$ $K^{y} \oplus Q^{z} \oplus B_{P}(1,2, \ldots n)$ olacak şekilde $x, y, z, n \in \mathbb{N}$ mevcut olmasidır.

\section{Materyal ve Metot}

$\mathrm{Bu}$ kısımda çalışmamızın temelini teşkil eden tanımları kurgulamamızı sağlayan temel kavramlara ve yer aldığı kaynaklara yer verilmiştir.

Tanım 1: $A$ bir modül olsun. $A$ nın radikali olan $\operatorname{Rad}(A)$ alt modülünü içeren her alt modül $A$ da tümleyene sahip ise $A$ ya güçlü tümlenmiş modül denir ve kısaca "srs-modül" șeklinde kısaltılır [9].

Tanım 2: Bir $A$ modülünün radikali (radikalini kapsayan her alt modülü) $A$ da direkt toplam terimi olacak şekilde tümleyene sahip ise $A$ ya (güçlü) $\bigoplus$ radikal tümlenmiş modül denir [10].

Güçlü $\oplus$-radikal tümlenmiş modüller için ilgili kaynakta $\bigoplus$-srs-modül kısaltması kullanılmıștır.

Tanım 3: Bir A modülünün $\delta$ - radikalinin her alt modülü $A$ da $\delta$ - tümleyene sahip ise $A$ ya güçlü genelleştirilmiş $\delta$-tümlenmiş modül denir ve kısaca " $\delta$ SGS modül" ile gösterilir [11].

Tanım 4: $A$ bir modül olsun. $\delta(A), A$ modülünde direkt toplam terimi olacak şekilde bir tümleyene sahip ise $A$ modülüne $\bigoplus-\delta$-radikal tümlenmiş modül denir.

Tanım 5: Bir $A$ modülünün $\delta(A)$ alt modülünü kapsayan her alt modülü $A$ da direkt toplam terimi olacak şekilde $\delta$-tümleyene sahip ise, $A$ ya güçlü $\bigoplus$ - $\delta$ radikal tümlenmiş modül denir.

\section{Bulgular}

Önerme 1: Her $\delta$-radikal modül (güçlü) $\bigoplus$ - $\delta$-radikal tümlenmiştir.

İspat: $A$ bir $\delta$-radikal bir modül olsun. Bu durumda $A=\delta(A)$ dır ve açık olarak $0 \leq A, \delta(A)$ nın $A$ da direkt toplam terimi olacak şekilde bir $\delta$ tümleyenidir. Dolayısıyla $A, \oplus-\delta$ - radikal tümlenmiş modüldür. Diğer taraftan $\delta(A)=A$ yani $\delta(A)$ alt modülünü içeren $A$ dan farklı bir alt modül yoktur. $\{0\}$ da $A$ nın direkt toplam terimi olacak şekilde $\delta$ tümleyeni olduğundan $A$ güçlü $\oplus-\delta$-radikal tümlenmiştir.

Önerme 2: $A$ bir modül olsun. $P_{\delta}(A)=\Sigma\{\mathrm{B} \leq$ $\mathrm{A} \mid \delta(\mathrm{B})=\mathrm{B}\} \leq \mathrm{A}$ (güçlü) $\oplus-\delta$-radikal tümlenmiştir.

İspat: $P_{\delta}(A), A$ nın en büyük $\delta$-radikal alt modülü olacağından Önerme 1 gereği iddia açıtır.

Yardımcı Teorem 3: $A$ (güçlü) $\oplus-\delta$-radikal tümlenmiş modül olsun. Bu takdirde $A, \delta$-radikal direkt toplam terimine sahiptir.

İspat: $A$ (güçlü) $\oplus$ - $\delta$-radikal tümlenmiş modül olsun. Bu takdirde $\delta(A)+B=A, \delta(A) \cap B=\delta(B) \ll_{\delta} B$ ve $A=B \oplus B^{\prime}$ olacak șekilde $B \leq A$ mevcuttur. Bu durumda $\delta(A)=\delta(B) \oplus \delta\left(B^{\prime}\right)$ olup $A=\delta(A)+B=$ $\left[\delta(B) \oplus \delta\left(B^{\prime}\right)\right]+B=B \oplus \delta\left(B^{\prime}\right)$ eşitliğinin her iki tarafinın $B^{\prime}$ ile arakesiti alınırsa $B^{\prime}=\delta\left(B^{\prime}\right)$ elde edilir.

Önerme 4: $A, P_{\delta}(A)=0$ koşulunu gerçekleyen bir modül olsun. Ĕger $A$ (güçlü) $\oplus$ - $\delta$-radikal tümlenmiş ise, $\delta(A) \ll_{\delta} A$ dır.

İspat : $A$ (güçlü) $\bigoplus$ - $\delta$-radikal tümlenmiș olduğundan $\delta(A)+B=A, \delta(A) \cap B=\delta(B) \ll_{\delta} B$ ve $A=B \oplus B^{\prime}$ olacak şekilde $B, B^{I} \leq A$ vardır. Yardımcı Teorem 3 den $\delta\left(B^{\prime}\right)=B^{\prime} \leq A \quad$ ve $P_{\delta}(A)=0$ olduğundan $\delta\left(B^{\prime}\right)=B^{\prime}=0$ olup $A=B$ elde edilir. Buradan $\delta(A) \ll_{\delta} A$ olur.

Önerme 5: $A, \delta(A) \ll A$ koşulunu gerçekleyen bir modül olsun. $\mathrm{Bu}$ takdirde $A \oplus$ - $\delta$-radikal tümlenmiş modüldür.

İspat: $\delta(A)+B=A$ olacak şekilde herhangi bir $B \leq$ $A$ için $\delta(A) \ll A$ olduğundan $B=A$ dır. Ayrıca $\delta(A) \cap B=\delta(A) \cap A=\delta(A) \ll A \quad$ ve $\quad A=$ 
$A \oplus 0$ olduğundan $A \oplus-\delta$-radikal tümlenmiş modüldür.

Sonuç 6: Her $\delta$-eşatomik modül $\oplus-\delta$ - radikal tümlenmiştir.

Teorem 7: $A_{1}, A_{2}, \ldots, A_{n}$ (güçlü) $\bigoplus-\delta$ - radikal tümlenmiş modül ise $A_{1} \oplus A_{2} \oplus \ldots \oplus A_{n}$ (güçlü) $\oplus-\delta$ radikal tümlenmiş modüldür.

İspat: $n=2$ için iddianın doğruluğunu göstermek yeterlidir. $A_{1}$ ve $A_{2} \delta$-radikal tümlenmiş modüller olmak üzere $A=A_{1} \oplus A_{2}$ olsun. Buradan $A=\delta(A)+$ $A_{1}+A_{2}$ yazilabilir. 0 halde, $\{0\}$ alt modülü $\delta(A)+$ $A_{1}+A_{2}$ nin $A$ da aşikar $\delta$-tümleyenidir. $A_{2} \cap$ $\left(\delta(A)+A_{1}\right) \leq A_{2}$ alt modülü için $A_{2} \cap\left(\delta(A)+A_{1}\right)=$ $A_{2} \cap\left[\left(\delta\left(A_{1}\right)+\delta\left(A_{2}\right)\right)+A_{1}=A_{2} \cap\left(\delta\left(A_{2}\right)+A_{1}\right)=\right.$ $\left(\delta\left(A_{2}\right)+A_{1}\right) \cap A_{2}=\delta\left(A_{2}\right)+\left(A_{1} \cap A_{2}\right)=\delta\left(A_{2}\right)$ olup $A_{2} \oplus$ - $\delta$ - radikal tümlenmiş olduğundan $\delta\left(A_{2}\right)+$ $B=A_{2}, \quad \delta\left(A_{2}\right) \cap B \ll_{\delta} B$ ve $A_{2}=B \oplus B^{\prime}$ olacak şekilde $B, B^{\prime} \leq A_{2}$ vardır. 0 halde $B, A_{2} \cap$ $\left(\delta(A)+A_{1}\right)$ in $A_{2}$ de direkt toplam terimi olacak şekilde $\delta$-tümleyenidir. 0 halde $B+0=B, \delta(A)+$ $A_{1}$ in $A$ da $\delta$-tümleyeni olur. Şimdi de benzer yolla $A_{1} \cap(\delta(A)+B)$ in $A_{1}$ de direkt toplam terimi olacak şekilde bir $\delta$-tümleyeninin varlığını gösterelim. Burada $\quad A_{1} \cap(\delta(A)+B)=A_{1} \cap\left(\delta\left(A_{1}\right)+\delta\left(A_{2}\right)+\right.$ $B)=\delta\left(A_{1}\right)+\left[A_{1} \cap\left(\delta\left(A_{2}\right)+B\right)\right]=\delta\left(A_{1}\right)+\left[A_{1} \cap\right.$ $\left.A_{2}\right]=\delta(A)$ ve $A_{1} \oplus-\delta$-radikal tümlenmiş olduğundan bir $C \leq A_{1}$ için $A_{1}=\delta\left(A_{1}\right)+C, \delta\left(A_{1}\right) \cap$ $C \ll_{\delta} C$ ve $A_{1}=C+C^{\prime}$ dür. 0 halde $C, A_{1} \cap(\delta(A)+$ $Y) \operatorname{nin} A_{1}$ de direkt toplam terimi olacak şekilde bir $\delta$ tümleyenidir. Sonuç olarak $B+C, \delta(A) \operatorname{nın} A$ da $\delta$ tümleyenidir. Ayrıca $\quad A=A_{1} \oplus A_{2}=$ $\left(C \oplus C^{\prime}\right) \oplus\left(B \oplus Y B^{\prime}\right)=(C \oplus B) \oplus\left(C^{\prime} \oplus B^{\prime}\right)$ olduğundan $C+B, A$ da bir direkt toplam terimidir. Buradan $A$, $\bigoplus$ - $\delta$-radikal tümlenmiștir.

Şimdi de $A_{1}$ ve $A_{2}$ güçlü $\bigoplus$ - $\delta$-radikal tümlenmiş modüller iken direkt toplamlarının da güçlü $\oplus$ - $\delta$ radikal tümlenmiş olduğunu gösterelim. $A=A_{1} \oplus A_{2}$ ve $B, A$ modülünün $\delta(A)$ alt modülünü kapsayan keyfi bir alt modülü olsun. Bu durumda $A=A_{1}+A_{2}+B$ yazılabilir. 0 halde $A_{1}+A_{2}+B,\{0\} \oplus-\delta$ tümleyenine sahiptir. $\delta\left(A_{2}\right) \leq \delta(A) \leq B \leq B+$ $A_{2}$ ve $\delta\left(A_{2}\right) \leq A_{2}$ olup $\delta\left(A_{2}\right) \leq A_{2} \cap\left(B+A_{1}\right)$ dir. Hipotez gereğince $A_{2}$ güçlü $\bigoplus$ - $\delta$-radikal tümlenmiş modül olduğundan $A_{2} \cap\left(B+A_{1}\right) \leq A_{2}, A_{2}$ de direkt toplam terimi olacak şekilde bir $C \delta$-tümleyenine sahiptir. Sonuç olarak $C \quad B+A_{1}$ için , $A$ da $\delta$-tümleyendir. Diğer taraftan $\delta\left(A_{1}\right) \leq \delta(A) \leq B \leq$ $B+C$ ve $\delta\left(A_{1}\right) \leq A_{1}$ olduğundan $\delta\left(A_{1}\right) \leq A_{1} \cap(B+$ $C)$ dir. Hipotez gereği $A_{1}$ güçlü $\delta$-radikal tümlenmiş olduğundan $A_{1} \cap(B+C), A_{1}$ de direkt toplam terimi olacak şekilde bir $D \delta$-tümleyenine sahiptir. Sonuç olarak $D+C, L \operatorname{nin} A$ da direkt toplam terimi olacak şekilde bir $\delta$-tümleyenidir. 0 halde $A$ güçlü $\oplus-\delta$ radikal tümlenmiş modüldür.

Yardımcı Teorem 8: $A$ bir modül $B, C \leq A$ olsun. $C \ll{ }_{\delta} A$ ve $B+C, A$ da bir $\delta$-tümleyene sahip ise $B, A$ da bir $\delta$-tümleyene sahiptir.
İspat: $X, B+C \operatorname{nin} A$ da bir $\delta$-tümleyeni olsun. $\mathrm{Bu}$ durumda $(B+C)+X=A$ ve $(B+C) \cap X \ll_{\delta} X$ dir. Buradan $(B+X)+C=A$ ve $C \ll_{\delta} A$ olduğundan $\exists C^{\prime} \leq C$ projektif yarı basit alt modülü için $(B+X) \oplus C^{\prime}=A$ dir. 0 halde, $B+\left(X \oplus C^{\prime}\right)=A$ olduğu açıktır. $B \cap\left(X \oplus C^{\prime}\right) \ll_{\delta} X \oplus C^{\prime}$ olduğunu gösterelim. $\quad B \cap\left(X \oplus C^{\prime}\right)=B \cap X \leq(B+C) \cap$ $X \ll_{\delta} X \leq X \oplus C^{\prime}$ olduğundan $B \cap\left(X \oplus C^{\prime}\right) \leq X \oplus C^{\prime}$ bulunur. Sonuç olarak, $X \oplus C^{\prime}, A$ da $B$ nin bir $\delta$-tümleyenir.

$\bigoplus-\delta$-tümlenmiş her modül güçlü $\oplus-\delta$-radikal tümlenmiştir. Önerme 9 ve Önerme 10 da özel şartlar altında tersinin de doğrulanabileceğini görmekteyiz.

Önerme 9: $A$ direkt toplam terimi özelliğine sahip güçlü $\oplus-\delta$-radikal tümlenmiş modül olsun. $\delta(A) \ll_{\delta} A$ ise $A$ bir $\bigoplus$ - $\delta$-tümlenmiş modüldür.

İspat: $B \leq A$ olsun. $B \leq B+\delta(A) \leq A$ ve $A$ güçlü $\bigoplus$ $\delta$ - radikal tümlenmiş modül olduğundan, $A=$ $(B+\delta(A))+U,(B+\delta(A)) \cap U \ll_{\delta} U$ ve $A=U \oplus U^{\prime}$ olacak şekilde $U, U^{\prime} \leq M$ vardır. Buradan Yardımcı Teorem 8 gereği projektif yarı basit bir $P^{\prime} \leq \delta(A)$ için $U \oplus P^{\prime}, B \operatorname{nin} A$ da bir $\delta$-tümleyenidir. Ayrıca $A=$ $U \oplus U^{\prime}$ ve $A=U \oplus\left(B+P^{\prime}\right)=(B+U) \oplus P^{\prime}$ ve $A$ direkt toplam terimi özelliğine sahip olduğundan $U \oplus P^{\prime}, A$ nın direkt toplam terimidir. Sonuç olarak $A$ bir $\bigoplus$ - $\delta$ - tümlenmiş modüldür.

Önerme 10: $A$ güçlü $\oplus$ - $\delta$-radikal tümlenmiş modül olsun. $\delta(A) \ll A$ ise $A \oplus-\delta$-tümlenmiş modüldür.

İspat: $B \leq A$ keyfi olsun. $B \leq B+\delta(A) \leq A$ ve $A$ güçlü $\bigoplus$ - $\delta$-radikal tümlenmiş modül olduğundan $A=$ $(B+\delta(A))+U,(B+\delta(A)) \cap U \ll_{\delta} U$ ve $A=U \oplus U^{\prime}$ olacak şekilde $U, U^{\prime}$ vardır. $A=(B+U)+\delta(A)$ ve $\delta(A) \ll A$ olduğundan $A=B+U$ olur. Diğer taraftan, $B \cap U \leq(B+\delta(A)) \cap U \ll_{\delta} U$ olduğundan $U, B \operatorname{nin} A$ da direkt toplam terimi olacak şekilde $\delta$-tümleyeni olur. Sonuç olarak $A \oplus$ - $\delta$-tümlenmiş modüldür.

Sonuç 11: $A, \delta$-eşatomik modül olsun. $A$ modülünün güçlü $\oplus$ - $\delta$-radikal tümlenmiş olması için gerekli ve yeterli koşul $A$ nın $\oplus$ - $\delta$-tümlenmiş olmasıdır.

Önerme 12: $A$ parçalanamaz ve $\delta(A) \neq A$ koşulunu gerçekleyen güçlü $\bigoplus-\delta$-radikal tümlenmiş modül olsun. Bu takdirde $\delta(A) \ll_{\delta} A$ dır.

İspat: $A$ güçlü $\oplus-\delta$-radikal tümlenmiş modül olduğundan $\delta(A)+B=A, \delta(A) \cap B \ll_{\delta} B$ ve $A=$ $B \oplus B^{\prime}$ olacak şekilde $B, B^{\prime} \leq A$ vardır. Ayrıca $A$ parçalanamaz olduğundan $B=0$ veya $B=A$ olmalıdır. $B=0$ ise $\delta(A)=A$ çelişkisi elde edilir. Bu durumda $\quad B=A \quad$ olmalidir. $\quad \delta(A) \cap$ $B \ll_{\delta} B$ için $\delta(B) \ll_{\delta} B$ olup $\delta(A) \ll_{\delta} A$ bulunur.

Aşağıdaki önerme güçlü $\oplus$ - $\delta$-radikal tümlenmiş bir modülün faktör modülünün de belli şartlar altında güçlü $\oplus-\delta$-radikal tümlenmiş modül olduğunu gösterir. 
Önerme 13: $A$ güçlü $\oplus$ - $\delta$-radikal tümlenmiş modül olsun. $B \leq A$ karakteristik alt modül ise $\frac{A}{B}$ bölüm modülü güçlü $\bigoplus$ - $\delta$-radikal tümlenmiştir.

İspat: $B \leq A$ karakteristik alt modülü ve $\frac{N}{B} \leq \frac{A}{B}$ faktör modülü; $\delta\left(\frac{A}{B}\right) \leq \frac{N}{B}$ olacak şekilde herhangi bir alt modülü olsun. $\alpha: A \rightarrow \frac{A}{B}$ doğal epimorfizması için $\alpha(\delta(A))=\frac{\delta(A)+B}{B} \leq \delta\left(\frac{A}{B}\right) \leq \frac{N}{B}$ olduğundan $\delta(A) \leq N$ olduğu kolayca görülür. $A$ güçlü $\oplus-\delta$-radikal tümlenmiş olduğundan $A=N+S, N \cap S \ll_{\delta} S$ ve $A=S \oplus S^{\prime}$ olacak şekilde $S, S^{\prime} \leq A$ vardır. Buna göre $S, N$ nin $A$ da $\delta$-tümleyeni olduğundan $\frac{S+B}{B}, \frac{N}{B} \operatorname{nin} \frac{A}{B}$ de $\delta$-tümleyeni olur. Ayrica $A=S \oplus S^{\prime}$ ve $B \leq A$ karakteristik alt modül olduğundan $B=(S \cap B) \bigoplus$ $\left(S^{\prime} \cap B\right)$ olur. Buradan $\frac{A}{B}=\frac{S \oplus B}{B} \oplus \frac{S^{\prime} \oplus B}{B} \quad$ olduğunu gösterelim. $\quad A=S \oplus S^{\prime} \Rightarrow A=S+S^{\prime} \Rightarrow \frac{A}{B}=\frac{S+B}{B}+$ $\frac{S^{\prime}+B}{B}$ olduğu açıktır. $B=(S \cap B) \oplus\left(S^{\prime} \cap B\right)$ olduğu göz önüne alınarak

$\frac{S+B}{B} \cap \frac{S^{\prime}+B}{B}=\frac{S+\left[(S \cap B)+\left(S^{\prime} \cap B\right)\right]}{B} \cap \frac{S^{\prime}+\left[(S \cap B)+\left(S^{\prime} \cap B\right)\right]}{B}=$ $\frac{S+\left(S^{\prime} \cap B\right)}{B} \cap \frac{S^{\prime}+(S \cap B)}{B}=\frac{\left[\left(S+\left(S^{\prime} \cap B\right)\right) \cap S^{\prime}\right]+(S \cap B)}{B}=$ $\frac{\left[\left(S^{\prime} \cap B\right)+\left(S \cap S^{\prime}\right)\right]+(S \cap B)}{B}=\frac{(S \cap B)+\left(S^{\prime} \cap B\right)}{B}=0$ olduğundan $\frac{S+B}{B} \leq{ }_{\oplus} \frac{A}{B}$ olup $\frac{A}{B}$ güçlü $\bigoplus-\delta$-radikal tümlenmiştir.

Sonuç 14: $A$ güçlü $\bigoplus$ - $\delta$-radikal tümlenmiş modül ise $\frac{A}{\delta(A)}$ yarı basittir.

İspat: $\delta(A) \leq A$ karakteristik alt modül olduğundan açıkça görülür.

Önerme 15: $A$ bir modül olsun. $\mathrm{Bu}$ takdirde $\delta\left(P_{\delta}(A)\right)=P_{\delta}(A)$ dir.

İspat: $\delta\left(P_{\delta}(A)\right) \leq P_{\delta}(A)$ olduğu açıktır. Tersine $a \in$ $P_{\delta}(A)$ olsun. $P_{\delta}(A)=\sum_{\substack{N \leq A \\ \delta(N)=N}} N$ olduğundan $a=$ $k_{i_{1}}+k_{i_{2}}+\cdots+k_{i_{n}} \quad$ olacak şekilde $k_{i_{j}} \in N_{i_{j}}(j=$ $1,2, \ldots, n) \quad$ vardır. $N_{i_{j}}=\delta\left(N_{i_{j}}\right)$ olduğundan $k_{i_{j}} \in$ $\delta\left(N_{i_{j}}\right)$ için $R_{k_{i_{j}}} \ll_{\delta} N_{i_{j}}$ dir. Buradan [5] (Lemma 1.2) gereği $\quad R a \ll_{\delta} N_{i_{1}}+N_{i_{2}}+\cdots+N_{i_{n}} \leq P_{\delta}(A) \quad$ olup $R a \ll_{\delta} P_{\delta}(A) \Rightarrow a \in \delta\left(P_{\delta}(A)\right)$ elde edilir. $\mathrm{Bu}$ ise $P_{\delta}(A) \leq \delta\left(P_{\delta}(A)\right)$ olması demektir. Sonuç olarak $\delta\left(P_{\delta}(A)\right)=P_{\delta}(A)$ olup $P_{\delta}(A), A$ nın en büyük $\delta$ radikal alt modülüdür.

Önerme 16: $A \oplus$ - $\delta$-radikal tümlenmiş modül ise $\frac{A}{P_{\delta}(A)} \bigoplus-\delta$-radikal tümlenmiştir.

İspat: $\alpha: A \rightarrow \frac{A}{P_{\delta}(A)}$ doğal homomorfizması için $\alpha(\delta(A)) \leq \delta\left(\frac{A}{P_{\delta}(A)}\right) \quad$ olacağından $\frac{\delta(A)+P_{\delta}(A)}{P_{\delta}(A)}=\frac{\delta(A)}{P_{\delta}(A)} \leq$ $\delta\left(\frac{A}{P_{\delta}(A)}\right)$ dır. $\delta\left(\frac{A}{P_{\delta}(A)}\right) \leq \frac{N}{P_{\delta}(A)} \leq \frac{A}{P_{\delta}(A)}$ için $\delta(A) \leq N$ ve
$A \oplus$ - $\delta$-radikal tümlenmiş olduğundan $\delta(A)+N=A$, $\delta(A) \cap N \ll_{\delta} N$ ve $A=N \oplus N^{\prime}$ dür. Bu durumda $\frac{N+P_{\delta}(A)}{P_{\delta}(A)}, \frac{\delta(A)}{P_{\delta}(A)}$ nin $\frac{A}{P_{\delta}(A)}$ de bir $\delta$ - tümleyeni olur. Ayrica, $\frac{A}{P_{\delta}(A)}=\frac{N+P_{\delta}(A)}{P_{\delta}(A)}+\frac{N^{\prime}+P_{\delta}(A)}{P_{\delta}(A)} \quad$ ve $\frac{N+P_{\delta}(A)}{P_{\delta}(A)} \cap$ $\frac{N^{\prime}+P_{\delta}(A)}{P_{\delta}(A)}=\frac{\left(N \cap N^{\prime}\right)+P_{\delta}(A)}{P_{\delta}(A)}=0$ olup $\frac{N+P_{\delta}(A)}{P_{\delta}(A)}, \frac{A}{P_{\delta}(A)}$ nin bir direkt toplam terimidir. Sonuç olarak, $\frac{A}{P_{\delta}(A)} \bigoplus-\delta$ radikal tümlenmiştir.

Önerme 17: $A$ güçlü $\oplus$ - $\delta$-radikal tümlenmiş modül ve $\frac{A}{\delta(A)}$ projektif olsun. Bu takdirde $\delta(A)$ nın $\oplus-\delta$ tümlenmiş olması için gerekli ve yeterli koşul $A$ nın $\bigoplus$ - $\delta$-tümlenmiş olmasıdır.

İspat: $(\Rightarrow): \frac{A}{\delta(A)}$ projekif olduğundan $0 \rightarrow \delta(A) \rightarrow$ $A \rightarrow \frac{A}{\delta(A)} \longrightarrow 0$ kisa tam dizisi parçalanabilirdir [1]. Bu durumda $A \cong \delta(A) \bigoplus \frac{A}{\delta(A)}$ yazılır. Dolayısıyla $B \cong$ $\frac{A}{\delta(A)}$ koşulunu sağlayan $B \leq A$ alt modülü için $A=$ $\delta(A) \oplus B$ yazılır. $A$ güçlü $\oplus-\delta$-radikal tümlenmiş modül olduğundan Sonuç 14 gereği $\frac{A}{\delta(A)} \cong B$ yarıbasittir. Dolayısıyla $B, \oplus-\delta$-tümlenmiştir. Hipotezden $\delta(A) \oplus-\delta$-tümlenmiş olduğundan, sonlu toplamları olan $A$ da $\bigoplus$ - $\delta$-tümlenmiş modüldür [8].

$(\Leftarrow): A \oplus-\delta$ - tümlenmiş modül olsun. $\delta(A) \leq A$ bir direkt toplam terimi ve karakteristik alt modülü olduğundan [12] (Teorem 2.9) gereği iddia açıktır.

Önerme 18 ve Teorem 21 de (güçlü) $\oplus$ - $\delta$-radikal tümlenmiş bir modülün belli özellikli alt modüllerinin de (güçlü) $\bigoplus-\delta$-radikal tümlenmiş olabileceği gösterilecektir.

Önerme 18: $A$ güçlü $\bigoplus$ - $\delta$-radikal tümlenmiş modül ve $C, A$ nın dual sonlu karakteristik alt modülü olsun. Eğer $C \leq_{\oplus} A$ ise $C$ güçlü $\oplus$ - $\delta$-tümlenmiş modüldür.

İspat: $L, C$ modülünün $\delta(C) \leq L$ olacak şekilde herhangi bir alt modülü olsun. $C \leq A$ dual sonlu ve direkt toplam terimi olduğundan $A=C \oplus U$ olacak şekilde sonlu üretilmiş $U \leq A$ vardır. $U$ sonlu üretilmiş olduğundan $\delta(U) \ll_{\delta} \mathrm{U}$ [13]. Ayrıca $A=$ $C \oplus U$ olduğundan $\delta(A)=\delta(C) \oplus \delta(U)$ olup $\delta(C) \leq$ $L \Rightarrow \delta(C)+\delta(U) \leq L+\delta(U) \Rightarrow \delta(A) \leq L+\delta(U) \leq$ $A$ olur. Buradan hipotez gereği $A$ güçlü $\oplus$ - $\delta$-radikal tümlenmiş modül olduğundan $(L+\delta(U))+N=A$, $(L+\delta(U)) \cap N \ll_{\delta} N$ ve $A=N \oplus N^{\prime}$ olacak şekilde $N, N^{\prime} \leq A \quad$ vardır. $\quad(L+N)+\delta(U)=A \quad$, $\delta(U) \ll_{\delta} A$ ise $\exists Y \leq \delta(U)$ projektif yarl basit alt modülü için $(L+N) \oplus Y=A \Rightarrow L+(N \oplus Y)=A \Rightarrow$ $[L+(N \oplus Y)] \cap C=A \cap C \Rightarrow L+[(Y \oplus N) \cap C]=$ $C \Rightarrow L+[(Y \cap C) \oplus(N \cap C)]=L+(N \cap C)=C \quad$ dir. (Çünkü $Y \leq \delta(U) \leq U$ ve $Y \leq U \Rightarrow Y \cap C \leq U \cap C \Rightarrow$ $Y \cap C=0)$. Buradan $L \cap(C \cap N)=L \cap N \leq(L+$ $\delta(U)) \cap N \ll_{\delta} N \Rightarrow L \cap(C \cap N) \ll_{\delta} A \quad$ dir. $C \leq A$ karakteristik alt modülü olduğundan $C=(C \cap N) \oplus$ 
$\left(C \cap C^{\prime}\right)$ dir. Ayrica, $L \cap(C \cap N) \leq A$ bir $\delta$ tümleyen, $L \cap(C \cap N) \leq C \cap N \leq A$ ve $C \cap N \leq_{\oplus} A$ olduğundan $L \cap(C \cap N) \ll_{\delta} C \cap N$ olur. $O$ halde $C$ güçlü $\oplus$ - $\delta$ - radikal tümlenmiştir.

Sonuç 19: $A$ güçlü $\bigoplus$ - $\delta$-radikal tümlenmiş ve $\delta(A), A$ nın dual sonlu ve direkt toplam terimi olacak şekilde bir alt modülü olsun. Bu takdirde $\delta(A)$ güçlü $\oplus-\delta$ radikal tümlenmiş olur.

Yardımcı Teorem 20: $A$ bir modül ve $\delta(A) \leq B \leq A$ olsun. $B \leq_{\oplus} A \Rightarrow \delta(A)=\delta(B)$ dir.

Ispat: $B \leq_{\oplus} A \Rightarrow A=B \oplus B^{\prime}$ olacak şekilde $\exists B^{\prime} \leq A$ vardır. Bu durumda $\delta(A)=\delta(B) \oplus \delta\left(B^{\prime}\right)$ olup $\delta(A) \cap$ $B=\left[\delta(B) \oplus \delta\left(B^{\prime}\right)\right] \cap B \Rightarrow \delta(A)=\delta(B) \oplus\left[\delta\left(B^{\prime}\right) \cap B\right]$ elde edilir. Buradan $\delta\left(B^{\prime}\right) \cap B \leq B^{\prime} \cap B=0 \Rightarrow$ $\left(\delta\left(B^{\prime}\right) \cap B\right)=0 \Rightarrow \delta(A)=\delta(B)$ bulunur.

Hatırlatmak gerekirse bir $A$ modülü, $A=A_{1}+A_{2}$ koşulunu sağlayan $A_{1}, A_{2} \leq_{\oplus} A$ için $A_{1} \cap A_{2} \leq_{\oplus} A$ oluyorsa $A$ modülü $(D 3)$ özelliğini sağlıyor denir.

Teorem 21: $A\left(D_{3}\right)$ koşulunu gerçekleyen güçlü $\bigoplus-\delta$ radikal tümlenmiş modül ve $B, A$ modülünün $\delta(A) \leq$ $B$ olacak şekilde bir alt modülü olsun. $B \leq_{\oplus} A$ ise $B$ güçlü $\oplus$ - $\delta$-radikal tümlenmiştir.

İspat: $\delta(B) \leq L \leq B$ olacak şekilde herhangi bir alt modül olsun. $\delta(A) \leq B$ ve $B \leq_{\oplus} A$ ise Yardımcı Teorem 20 gereği $\delta(A)=\delta(B)$ olup $\delta(A) \leq L$ olur. $A$ güçlü $\oplus$ - $\delta$-radikal tümlenmiş olduğundan, $A=L+$ $N, L \cap N \ll_{\delta} N$ ve $A=N \oplus N^{\prime}$ olacak şekilde $N, N^{\prime} \leq$ $A$ vardır. $A=L+N$ için modüler kuraldan $B=A \cap$ $B=(L+N) \cap B=L+(N \cap B)$ dir. Ayrica $A=B+$ $N, B \leq_{\oplus} A$ ve $N \leq_{\oplus} A$ olup $A\left(D_{3}\right)$ özelliğine sahip olduğundan $B \cap N \leq_{\oplus} A$ dir. Bu durumda bir $X \leq A$ için $A=(B \cap N) \oplus X$ dir. $L \cap(B \cap N) \leq L \cap N \ll_{\delta} N$ olduğundan $L \cap(B \cap N) \ll_{\delta} A$ dır. Ayrıca $B \cap$ $N \leq_{\oplus} A$ oduğundan $L \cap(B \cap N) \ll_{\delta} B \cap N$ dir. Sonuç olarak $B \cap N, L \operatorname{nin} B$ de bir $\delta$-tümleyenidir. Ayrıca, Modüler kural gereği $B \leq A$ için $A=(B \cap N) \oplus X$ iken $B=(B \cap N) \oplus(B \cap X)$ olup $B \cap N \leq_{\oplus} A$ dır. Dolayısıyla $B$ güçlü $\oplus-\delta$-radikal tümlenmiş modüldür.

Teorem 22: $A$ parçalanamaz modül olsun. $A$ güçlü $\bigoplus$ - $\delta$-radikal tümlenmiş modül ise $\delta(A)=A$ ya da $A$ $\delta$-lokaldir.

İspat: Kabul edelim ki $\delta(A) \neq A$ olsun. Bu takdirde $\frac{A}{C}$ tekil olacak şekilde $\exists C \leq A$ maksimal alt modülü vardır. $A$ güçlü $\bigoplus-\delta$-radikal tümlenmiş modül ve $\delta(A) \leq C$ olduğundan $A=C+N, C \cap N \ll_{\delta} N$ ve $A=$ $N \oplus N^{\prime}$ olacak şekilde $N, N^{\prime} \leq A$ vardır. $C \leq A$ maximal ve $N, C \operatorname{nin} A$ da $\delta$-tümleyeni olduğundan [14] (Lemma 2.22) gereği $N \delta$-lokal ya da projektif yarı basittir. $A$ parçalanamaz modül olduğundan $A=$ $N \oplus N^{\prime}$ için $N=0$ veya $N=A$ dır. $N=0 \Rightarrow A=C$ bulunur ki bu durum $C$ nin maksimalliği ile çelişir. 0 halde $N=A$ olmalıdır. Bu durumda $A \delta$-lokal ya da projektif yarı basittir. $\delta(A) \neq A$ olduğundan $A$ projektif yarı basit olamaz. $A \delta$-lokaldir.

Önerme 23: $A$ direkt toplam terimi özelliğine sahip bir modül olsun. $A$ güçlü $\oplus-\delta$-radikal tümlenmiş modül ise $A \oplus$ - $\delta$-dual sonlu tümlenmiştir.

İspat: Teo 2.7 [12] gereği $A$ modülü $D T T$ özelliğine sahip olduğundan $A$ nin her $L$ maksimal alt modülünün $A$ da direkt toplam terimi olacak şekilde bir $\delta$-tümleyeninin varlığını göstermek yeterlidir. $\forall L \leq A$ maksimal alt modülü için $\delta(A) \leq L \quad \mathrm{~V}$ $\delta(A) \not L . \quad \delta(A) \leq L$ ise $A$ güçlü $\oplus-\delta$-radikal tümlenmiş modül olduğundan $L, A$ da direkt toplam terimi olacak şekilde bir $\delta$-tümleyene sahiptir. $\delta(A) \not L$ ise $\exists a \in \delta(A) \quad$ için $\quad a \notin L \operatorname{dir} . L \leq A$ maksimal olduğundan $L+R a=A \quad$ dır. $\quad a \in$ $\delta(A)$ olduğundan $R a \ll_{\delta} A$ olur. Bu durumda $\exists D \leq$ $R a$ projektif yarı basit alt modülü için $L \oplus D=A$ olup $D, L$ nin $A$ da direkt toplam terimi olacak şekilde $\delta$-tümleyenidir. Sonuç olarak $A \oplus-\delta$-dual sonlu tümlenmiştir.

Önerme 24: $A$ bir modül ve $\delta(A) \leq A$ dual sonlu alt modülü olsun. $A, \oplus$ - $\delta$-dual sonlu tümlenmiş modül ise $A$ güçlü $\oplus$ - $\delta$-radikal tümlenmiştir.

İspat: $\quad B, A \quad$ modülünün $\quad \delta(A) \leq B \quad$ koşulunu gerçekleyen keyfi bir alt modülü olsun. $\frac{A}{\delta(A)}$ sonlu üretilmiş olduğundan $\frac{A / \delta(A)}{B / \delta(A)} \cong \frac{A}{B}$ sonlu üretilmiş olur. Hipotez gereği $A, \oplus-\delta$-dual sonlu tümlenmiș olduğundan $B, A$ modülünde direkt toplam terimi olacak şekilde $\delta$-tümleyene sahiptir. Dolayısıyla $A$ güçlü $\oplus$ - $\delta$-radikal tümlenmiştir.

Önerme 23 ve Önerme 24 gereği aşağıdaki sonuç verilebilir.

Sonuç 25: $A$ eş modül ve $\delta(A) \leq A$ dual sonlu alt modülü olsun. $A$ modülünün güçlü $\oplus-\delta$ - radikal tümlenmiş olması için gerekli ve yeterli koşul $A$ modülünün $\bigoplus$ - $\delta$-dual sonlu tümlenmiş olmasıdır.

Önerme 26: $A$ parçalanamaz modül olsun. $\mathrm{Bu}$ takdirde $A$ nın $\oplus-\delta$ - radikal tümlenmiş olması için gerekli ve yeterli koşul $\delta(A) \ll_{\delta} A$ veya $\delta(A)=A$ olmasidır.

İspat: $A$ parçalanamaz modülü $\oplus-\delta$-radikal tümlenmiş olsun. Bu durumda $\delta(A)+B=A, \delta(A) \cap$ $B \ll_{\delta} B$ ve $A=B \oplus B^{\prime}$ olacak şekilde $\exists B, B^{\prime} \leq A$ vadır. $A$ parçalanamaz olduğundan $B=0 \quad \vee \quad B=A$ dır. Ĕ̆er $B=0 \Rightarrow \delta(A)=A$ dır. Ĕger $B=$ $A$ ise $\delta(A) \cap B=\delta(B)=\delta(A) \ll_{\delta} A \quad$ dir. Tersine $\delta(A)=A$ olsun. Bu durumda $A \delta$-radikal modül olacağından Önerme 1 gereği iddia açıktır. $\delta(A) \ll_{\delta} A$ olsun. Bu durumda $\delta(A)+L=A$ olacak şekilde bir $L \leq A$ için hipotez gereği $H \oplus L=A$ olacak șekilde 
projektif yarı basit bir $H \leq \delta(A)$ vardır. $A$ parçalanamaz olduğundan $H=0$ veya $H=A$ dır. $H=0 \Rightarrow L=A, \delta(A)$ nin $A$ da direkt toplam terimi olacak şekilde $\delta$-tümleyenidir. $H=A$ ise $L=0 \Rightarrow A=$ $\delta(A)$ olup Önerme 1 gereği $A, \oplus-\delta$-radikal tümlenmiştir.

Aşağıdaki önermede güçlü $\bigoplus$ - $\delta$-radikal tümlenmiş modüllerin $\delta$-yarı mükemmel halkalar üzerinde bir karakterizasyonu verilmektedir.

Önerme 27: $R$ direkt toplam terimi özelliğine sahip bir halka olsun. $R$ nin $\delta$-yarı mükemmel halka olması için gerekli ve yeterli koşul her sonlu üretilmiş serbest $R$-modülünün güçlü $\bigoplus-\delta$-radikal tümlenmiş olmasidır.

İspat: $(\Rightarrow): I=\{1,2, \ldots, n\}$ bir indis kümesi ve $F=$ $R^{(I)}$ sonlu üretilmiş serbest $R$-modül olsun. $R, \delta$-yarı mükemmel olduğundan [8] (Lemma 3.5) gereği $\oplus-\delta$ tümlenmiştir. Dolayısıyla ${ }_{\mathrm{R}} \mathrm{R}$ güçlü $\oplus-\delta$-radikal tümlenmiş modüldür. Sonlu sayıda güçlü $\oplus-\delta$-radikal tümlenmiş modülün toplamıda güçlü $\oplus-\delta$-radikal tümlenmiş olduğundan $F$ için iddia doğrulanır.

$(\Leftarrow)$ : Her sonlu üretilmiş serbest $R$-modül güçlü $\bigoplus$ - $\delta$ radikal tümlenmiş olsun. $\mathrm{Bu}$ durumda ${ }_{\mathrm{R}} \mathrm{R}$ güçlü $\oplus-\delta$ - radikal tümlenmiştir. ${ }_{\mathrm{R}} \mathrm{R}$ sonlu üretilmiş olduğundan $\delta(R) \ll_{\delta} R$ olup Önerme 2 gereği $\quad{ }_{\mathrm{R}} \mathrm{R} \oplus$ - $\delta$-tümlemiştir. [8] (Lemma 3.5) gereği $R \delta$-yarı mükemmel halkadır.

Güçlü $\oplus$-radikal tümlenmiş her halka $\oplus$ - $\delta$-radikal tümlenmiştir. Aşağıda tersinin her zaman gerçeklenemeyeceği bir örnekle doğrulanmaktadır.

Örnek: $T=\prod_{i}^{\infty} \mathbb{Z}_{2}, R=<\bigoplus_{i=1}^{\infty} \mathbb{Z}_{2}, 1_{T}>\leq R$ olsun $R$ regüler olan ancak yarı mükemmel olmayan bir halka örneğidir. $\mathrm{Bu}$ durumda [15] (Cor. 2.4) gereği $R$ üzerinde güçlü $\bigoplus$-radikal tümlenmiş olmayan sonlu üretilmiş serbest bir $A$ modülü mevcuttur. Diğer yandan regüler $R$ halkası üzerindeki her serbest modül regüler olduğundan ${ }_{\mathrm{R}} \mathrm{R}$ regülerdir [15]. Dolayısıyla direkt toplam terimi özelliğine sahiptir. Diğer yandan ${ }_{\mathrm{R}} \mathrm{R} \delta$ - yarı mükemmel halka olduğundan Önerme 27 gereği $A R$-modülü güçlü $\bigoplus$ $\delta$-radikal tümlenmiştir.

$R$ bir Dedekind Bölgesi ve $D, R$ nin sıfırdan farklı bir ideali olsun. $n \in \mathbb{N}$ olacak şekilde $K_{D}(1,2, \ldots n)$ notasyonu ile $\frac{R}{D}, \frac{R}{D^{2}}, \ldots, \frac{R}{D^{n}}$ kopyalarının direkt toplamı gösterilecektir.

Önerme 28: $R$ ayrık değerlendirme halkası ve $A$, $\operatorname{Rad} A \ll A$ olacak şekilde bir $R$-modül olsun.

1. $A \oplus$-tümlenmiştir.

2. $A$ güçlü $\bigoplus$-radikal tümlenmiştir.

3. $A$ güçlü $\oplus$ - $\delta$-radikal tümlenmiştir.

4. $A \oplus$ - $\delta$-tümlenmiștir.
5. $x, y, z$ ve $n$ doğal sayılar olmak üzere $A=R^{x} \bigoplus$ $K^{y} \oplus Q \oplus B_{P}(1,2, \ldots, n)$ şeklindedir.

İspat: $(1) \Rightarrow(2) \Longrightarrow(3)$ tanımlar gereği açıktır. (3) $\Rightarrow$ (4) Önerme 10 dan açıktır. (4) $\Rightarrow$ (5) $\Rightarrow$ (1) [12] (Teorem 5.4) den açıktır.

Örnek: $\mathbb{Z}$-modül $\mathbb{Z}$ modülünün $\delta$-eşatomik modül olduğu [16] (Example 2.19(3)) den görülebilir. Ayrıca [16] (Lemma 2,3) gereği, $\delta(\mathbb{Z}) \ll \mathbb{Z}$ olduğu da göz önüne alınırsa Önerme 5 gereği $\mathbb{Z}$-modül $\mathbb{Z}$ nin $\bigoplus-\delta$ radikal tümlenmiş modül olduğu doğrulanır. İlaveten, bu örneğin Önerme 4 ü de somutlaștırdığı görülebilir.

\section{Tartışma ve Sonuç}

Bu çalışmada kurgulanan tanımların ıșığında, verilen bir $A$ modülü için aşağıdaki diyagram elde edilir. Güçlü $\bigoplus$-radikal tümlenmiș modül $\Longrightarrow$ güçlü $\oplus-\delta$ radikal tümlenmiş modül $\Longrightarrow \oplus$ - $\delta$-radikal tümlenmiş modül $\bigoplus$-srs-modüllerin sınıfı ile güçlü $\oplus$ - $\delta$-radikal tümlenmiş modüllerin sınıfı arasındaki kapsamanın öz olmasının vurgulanmasının yanısıra ayrık değerlendirme halkası üzerinde $\operatorname{Rad}(A) \ll A$ koşulunu gerçekleyen $M$ modülleri için bu kavramların denkliğinin gösterilmesi oldukça önemlidir.

\section{Teşekkür}

$\mathrm{Bu}$ çalışmanın geliştirilmesine ve değerlendirilmesine katkı sağlayan değerli hakemlere teşekkürü bir borç biliriz.

\section{Etik Beyanı}

Bu çalışmada, "Yükseköğretim Kurumları Bilimsel Araștırma ve Yayın Etiği Yönergesi" kapsamında uyulması gerekli tüm kurallara uyulduğunu, bahsi geçen yönergenin "Bilimsel Araştırma ve Yayın Etiğine Aykırı Eylemler" başlığı altında belirtilen eylemlerden hiçbirinin gerçekleştirilmediğini taahhüt ederiz.

\section{Kaynakça}

[1] Wisbauer, R. 1991. Foundations of Module and Ring Theory. Revised and Updated English Edition, Gordon and Breach, Philedelphia

[2] Zöschinger, H. 1975. Moduln die in jeder Erweiterung ein Komplement Haben. Mathematica Scandinavica, 35, 267-287

[3] Zöschinger, H. 1974a. Komplementierte Moduln über Dedekindringen. Journal of Algebra, 29, 4256.

[4] Mohamed, S.H., Müller, B.J. 1990. Continuous and Discrete Modules. London Mathematical Society Lecture Note Series, Cambridge University Press, Cambridge, UK

[5] Zhou, Y. 2000. Generalizations of Perfect, Semiperfect and Semiregular Rings. Algebra Colloquium, 7(3), 305-318. 
[6] Sözen, Ö. E., Eren, Ş. 2018. Modules That Have a Generalized $\delta$-Supplement in Every Cofinite Extension. JP Journal of Algebra Number Theory and Applications, 40(3).

[7] Koşan, M. T. 2007. $\delta$-Lifting and $\delta$-Supplemented Modules. Algebra Colloquium, 14(1), 53-60.

[8] Talebi, Y., Pour, M. H. 2009. On $\oplus$ - $\delta$ Supplemented Modules. Journal of Algebra Number Theory: Advances and Applications, 1(2), 89-97.

[9] Büyükaşık, E., Türkmen, E. 2012. Strongly Radical Supplemented Modules. Ukrainian Mathematical Journal, 63(8), 1306-1313.

[10] Türkmen, B. N., Pancar, A. 2013. Strongly Radical $\oplus$-Supplemented Modules. Ukrainian Mathematical Journal, 65(4), 612-622.

[11] Eryılmaz, F. 2017. Stronglt Generalized (Weakly) $\delta$-Supplemented Modules. Vasile Alecsandri
University of Bacau, Faculty of Sciences, Scientific Studies and Research, Series Mathematics and Informatics, 27(1), 21-32.

[12] Tribak, R., Talebi. Y., Hamzekolaei, A.R.M., Asgari, S. 2016. $\oplus$-Supplemented Modules Relative to an Ideal, Hacettepe Journal of Mathematics and Statistics, 45(1), 107-120.

[13] Tribak, R. 2012. Finitely Generated $\delta$ Supplemented Modules are Amply $\delta$. Supplemented. Bulletin of Australian Mathematical Society, 86, 430-439.

[14] Tribak, R. 2013. On $\delta$-Local Modules and Amply $\delta$-Supplemented Modules. Journal of Algebra and Its Applications, 12(2), 1250144.

[15] Lee, G., Rizvi, T. S., Roman, C. 2013. Modules Whose Endomorphism Rings are Von-Neuman Regular. Communications Algebra, 41(11), 4066-4088. 